\title{
SISTEMA DE INSPEÇÃO INTEGRADA PARA CONTROLE DOS ATIVOS NA ARCELORMITTAL TUBARÃO *
}

\author{
Antonio Carlos Aguiar Gagno Junior ${ }^{1}$ \\ Jorge Pires ${ }^{2}$ \\ Alexandre Vellozo dos Santo ${ }^{3}$
}

\section{Resumo}

Este trabalho tem como objetivo mostrar a plataforma de integração de dados de inspeção preditiva e sensitiva desenvolvida na ArcelorMittal Tubarão para controle da condição dos ativos, com foco em ganho de produtividade, confiabilidade, e de eficácia das avaliações. O trabalho se constitui no desenvolvimento um sistema informatizado que integre e disponibilize dados em uma plataforma única, a partir de dados migrados dos diversos sistemas específicos já existentes na ArcelorMittal Tubarão, como é o caso do sistema de controle de vibrações, lubrificantes, termografia, grandezas elétricas, além dos dados de inspeção sensitiva. O sistema visa integrar os dados dos ativos das unidades operacionais da ArcelorMittal Tubarão que tenham variáveis de controle, possibilitando a correlação entre estas variáveis de condição e também com as variáveis de processo. A integração dos dados auxilia as equipes de manutenção na detecção imediata e direta das condições operacionais dos ativos, bem como permite avaliar antecipadamente a sua probabilidade de falhas. O sistema também permite construir uma base de dados consistente, que será um pilar importante para desenvolvimento de sistema de auxílio na tomada de decisão.

Palavras-chave: Produtividade, manutenção Preditiva, Análise de Dados.

\section{INTEGRATED INSPECTION SYSTEM FOR ASSET MANAGEMENT AT ARCELORMITTAL TUBARÃO}

\section{Abstract}

This work aims to show the data integration platform of predictive and sensitive inspection developed at ArcelorMittal Tubarão to control the condition of the assets, focusing on productivity gain, reliability, and evaluation effectiveness. The work consists in the development of a computerized system that integrates and makes available data on a single platform, from data migrated from the various specific systems already existing in ArcelorMittal Tubarão, such as the vibration control system, lubricants, thermography, magnitudes Electrical, in addition to sensitive inspection data. The system aims to integrate the data of the assets of the ArcelorMittal Tubarão operating units that have control variables, allowing the correlation between these condition variables and also the process variables. Data integration assists maintenance teams in the immediate and direct detection of asset operating conditions, as well as enables them to evaluate their probability of failure in advance. The system also allows the construction of a consistent database, which will be an important pillar for the development of a decision support system.

Keywords: Productivity, Predictive Maintenance; Data Analysis.

1 Graduado Engenharia Elétrica, Mestre em Gerenciamento de Projetos, Especialista na área de Engenharia de Automação de Processos, ArcelorMittal Tubarão, Vitória, Espírito Santo, Brasil.

2 Graduado em Administração, Especialista na área de Tecnologia da Informação, ArcelorMittal Tubarão, Vitória, Espírito Santo, Brasil.

3 Graduado em Engenharia Mecânica, Especialista na área de Engenharia de Manutenção, ArcelorMittal Tubarão, Vitória, Espírito Santo, Brasil. 


\section{INTRODUÇÃO}

A ArcelorMittal Tubarão é uma indústria de produção de aço localizada na Ponta de Tubarão no Município de Serra no ES, e iniciou sua operação em 1983.

A Figura 1 apresenta uma vista panorâmica da usina.

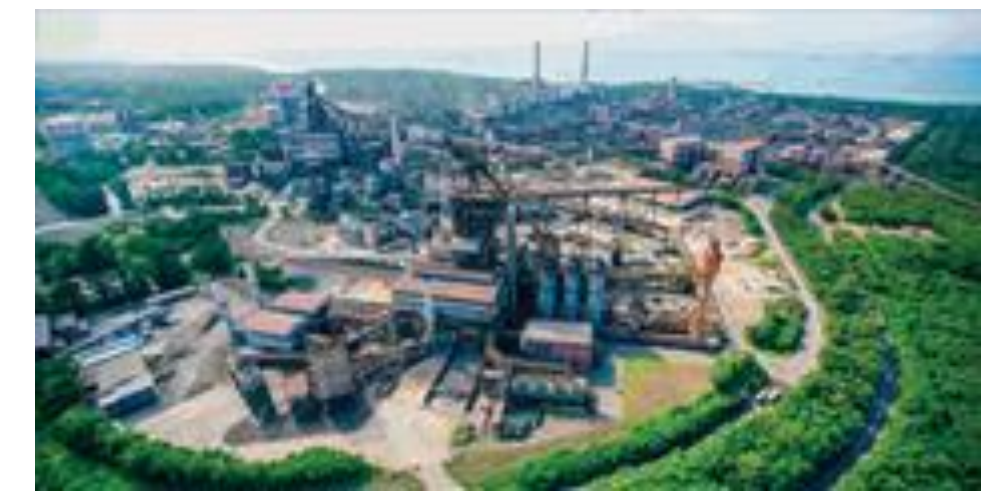

Figura 1 - Vista panorâmica da Usina Siderúrgica ArcelorMittal Tubarão

\section{1- O Papel da Manutenção}

A Manutenção da ArcelorMittal Tubarão tem como principal objetivo a estabilidade operacional da planta. Tal atitude traduz os ganhos gerados na produção e nos resultados financeiros, além dos consequentes benefícios em outras áreas de extrema importância para o grupo, como segurança, sustentabilidade e meio ambiente.

A manutenção tem como foco a confiabilidade dos equipamentos. Desta forma, o grupo conta com equipes capacitadas para atuarem na manutenção de forma preventiva, buscando o desenvolvimento das técnicas e metodologias de forma continua, para melhorar a eficiência e qualidade do serviço. Além disso, o trabalho das equipes de manutenção acontece de forma integrada às equipes de produção, visando a estabilidade operacional com segurança e eficiência.

A metodologia utilizada pela manutenção é baseada em quatro pilares: Inspeção dos equipamentos; Planejamento, programação e controle; Manutenção por conjunto; Execução das ações.

Todas as áreas de manutenção da usina são suportadas por uma Gerência de Tecnologia e Confiabilidade de Manutenção que tem como principal objetivo dar suporte no que se refere manutenção e gestão de ativos, promovendo melhorias contínuas nos processos de manutenção da ArcelorMittal Tubarão. Dentro das principais atividades desenvolvidas, destacam-se:

- Definir e disseminar políticas e modelo para manutenção e gestão de ativos.

- Implantar e gerenciar a Manutenção Centrada em Confiabilidade (RCM), técnicas de confiabilidade quantitativa e consultoria técnica na área de confiabilidade, em equipamentos e sistemas críticos.

- Dar suporte as áreas de manutenção na execução e diagnose de todas as técnicas ligadas à preditiva eletro-mecânica, além de consultoria na criação de planos e métricas. 
- Implementar, treinar, controlar e auditar os executantes, na aplicação e uso das Técnicas de Manutenção Preditiva, CMMS, NR's, ARM, entre outras.

- Realizar consultorias proativas para melhoria da manutenção com base em auditorias de cumprimento de planos, política, registros e indicadores.

\section{2- Inspeção e Controle dos Ativos na ArcelorMittal}

Na ArcelorMittal Tubarão se aplicam as melhores práticas de inspeção e controle da condição dos equipamentos, partindo do controle básico realizado através da inspeção sensitiva, e se estendendo às diversas técnicas de controle preditivo.

Os itens de controle sensitivos são gerenciados diretamente no Sistema Informatizado de manutenção (CMMS) denominado de SISMANA. Tal sistema foi desenvolvido internamente na empresa e abrange toda a gestão da manutenção (inspeção e serviço). Já as técnicas de preditivas específicas como analises de vibrações, analises de lubrificantes, termografia, são gerenciadas em sistemas especializados, e nesses casos as informações ficam restritas a estes aplicativos.

Este cenário faz com que grande parte das informações geradas, referente à condição dos equipamentos, fiquem disponíveis de forma difusa, o que dificulta o trabalho dos especialistas e técnicos durante as avaliações e tomadas de decisão no âmbito da manutenção.

O grupo DE TRABALHO CRIADO PARA buscou conhecer os sistemas disponíveis no mercado que atendessem as necessidades levantadas. Constatou-se que as soluções disponíveis no mercado apresentavam plataformas especializadas e com maior foco na monitoração on-line (monitoração contínua) de equipamentos críticos e complexos. Dessa forma, o investimento nesse tipo de sistema para a manutenção não era justificado, visto a pequena quantidade de ativos que seriam contemplados. Outros sistemas disponíveis no mercado, aplicáveis ao caso do grupo, com monitoração offline (monitoração periódica), também não se mostraram adequados devido às incompatibilidades com os sistemas proprietários da AMT além da exigência de complexas customizações. É importante ressaltar que a preservação dos sistemas de controle preditivos já existentes na AMT foi uma das premissas do trabalho. Isso se justifica por razoes técnicas e econômicas, onde foram considerados os bancos de dados com histórico de mais de 14 anos, e os altos investimentos realizados na aquisição desses sistemas.

Baseado nas considerações feitas acima, o grupo decidiu pelo desenvolvimento interno de um sistema de integração de informação das plataformas já existentes na AMT.

\section{2- PLATAFORMA DE INTEGRACAO DE INSPEÇÃO}

A plataforma de inspeção integrada desenvolvida na ArcelorMittal Tubarão foi a solução encontrada pelo grupo para os problemas levantados no capitulo anterior. Esse sistema é aplicável a todos os ativos da planta e tem como principal objetivo integrar os dados das inspeções preditivas e sensitivas em uma plataforma única, proporcionando um aumento de produtividade das equipes de inspeção e 
confiabilidade na avaliação prévia dos equipamentos. Entre os objetivos e benefícios dessa plataforma para a manutenção, se espera com o desenvolvimento:

- Integrar as técnicas preditivas em uma plataforma única.

- Fazer a gestão dos equipamentos em alarme em um único sistema.

- Fazer a gestão dos indicadores da manutenção preditiva de forma otimizada.

- O aumento da produtividade dos técnicos de inspeção na avaliação prévia dos equipamentos.

- Uso de uma plataforma sem limitação de usuários com uma interface amigável.

Outro ponto de destaque da plataforma é que ela foi concebida para integração de variáveis com monitoração off-line (monitoração periódica), visando alcançar além dos equipamentos de criticidade alta (AA e A) também os equipamentos de menor criticidade (B e $C$ ), visto que estes representam mais de $70 \%$ dos ativos da usina, 0 que tem impacto muito significativo nos custos da manutenção e visto também a existência de plataforma on-line (PIMS).

\section{1- $\quad$ Arquitetura do Sistema}

Conforme mencionado no capitulo anterior, o sistema teve como premissa a preservação das plataformas especializadas já utilizadas na ArcelorMittal Tubarão, além do sistema próprio de gestão de manutenção CMMS (Simana). Assim, o desenvolvimento do sistema foi idealizado conforme a arquitetura apresentada na Figura 2, onde uma plataforma integradora deveria absorver as informações de todos os outros bancos de dados. O ponto chave do trabalho seria o desenvolvimento de conectores com a finalidade de promover a comunicação dos sistemas e a plataforma de integração. Além disso, seria estruturada uma interface de visualização para exibição dessas informações.

Com a infraestrutura do projeto pensada e estruturada, foi preciso trabalhar na correlação das informações dos sistemas. Cada sistema apresentado na Figura 2 possui sua própria nomenclatura de identificação dos ativos controlados

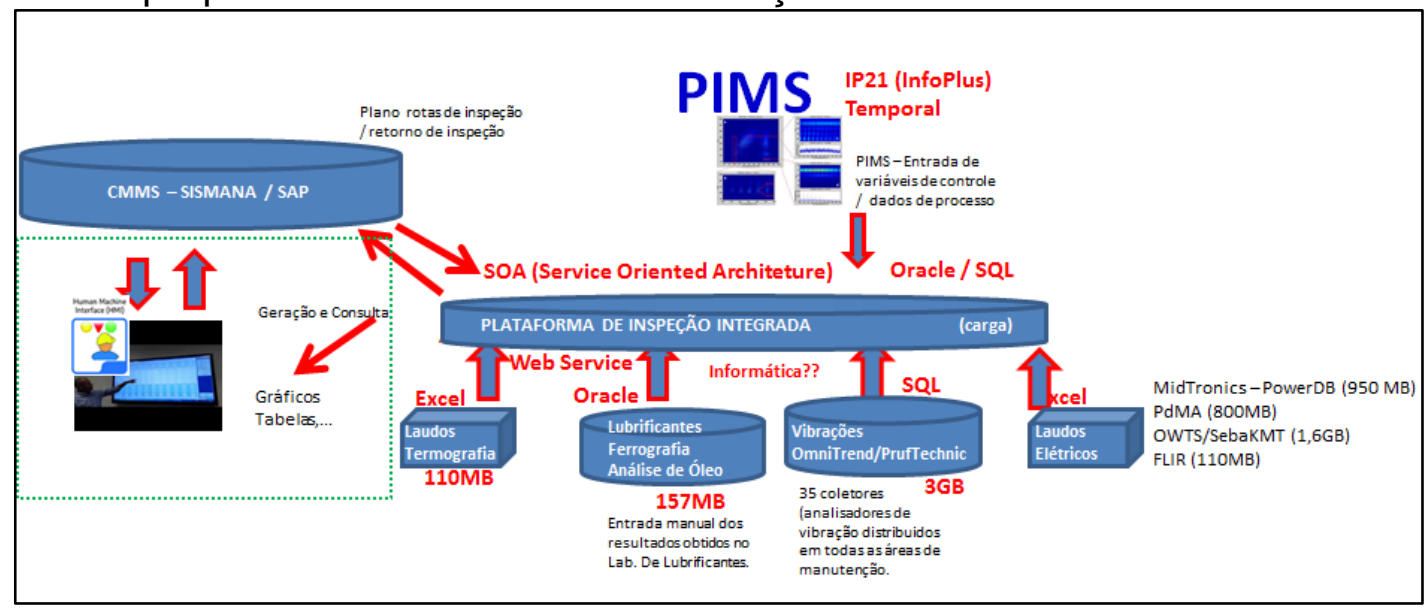

Figura 2 - Arquitetura idealizada para a Plataforma de Integração dos dados.

Como o sistema CMMS abrange a grande maioria dos ativos da usina, buscou-se utilizar a sua nomenclatura neste trabalho de correlação das informações. Dessa forma, foi possível reunir os dados de diferentes fontes em um único sistema. 
Devido ao imenso numero de ativos existentes na AMT, foram utilizados alguns critérios durante a escolha dos equipamentos que fariam parte do cadastro da plataforma de integração. Desses critérios podemos citar:

- Criticidade ao nível hierárquico de equipamento;

- Revisão dos planos de inspeção de acordo com metodologia do RCM;

- Com informação oriunda de diferentes sistemas;

- Outras.

O trabalho de correlação das informações foi realizado em paralelo com a equipe responsável por revisar os planos de inspeção dos ativos com base na metodologia $\mathrm{RCM}$. Esses planos revisados foram utilizados para identificar as variáveis de controle de cada ativo que deveriam estar presentes na plataforma de integração. Além disso, buscou-se também o apoio das equipes responsáveis pelas técnicas preditivas para identificar as variáveis de controle existentes para cada ativo.

Este trabalho de levantamento de dados e correlação foi primeiramente realizado em uma área piloto e prosseguiu para outras áreas da usina. Durante os primeiros testes foram identificados alguns problemas com a infraestrutura criada para o projeto. Dessa forma, foram feitas algumas mudanças na arquitetura original da plataforma. Foi utilizado um banco de dados Oracle dedicado e uma interface de visualização em ambiente web. A arquitetura atual pode ser vista na Figura 3.

Atualmente o sistema possui controle de ativos de seis diferentes áreas da AMT. Com isso, já foram cadastrados cerca de 1600 ativos e mais de 20000 variáveis de controle.

O projeto possui um longo horizonte de trabalho. As atividades serão expandidas pelas demais áreas da usina, com uma estimativa de cadastro de mais de 15000 ativos, e novas ferramentas continuarão em desenvolvimento na interface da plataforma de integração.

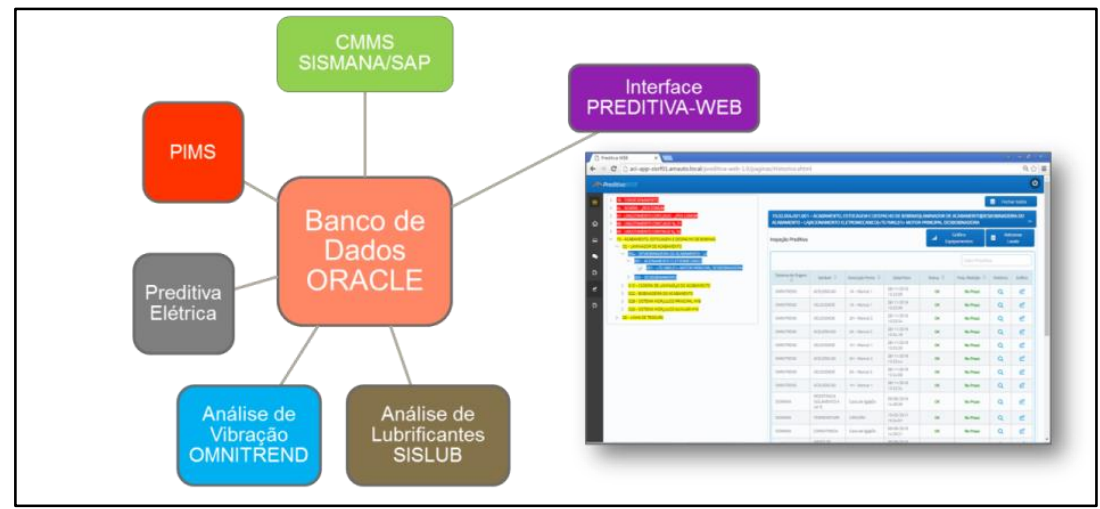

Figura 3 - Arquitetura atual da plataforma de integração de dados.

\section{3- PLATAFORMA DE INTEGRAÇÃO DE INSPEÇÃO - PreditivaWeb}

Preditiva WEB é uma interface de exibição das informações levantadas e cadastradas na plataforma de integração mencionada no capitulo anterior. Sua 
finalidade é proporcionar um ambiente virtual com ferramentas que auxiliem os técnicos e especialistas durante avaliação das condições de um ativo.

Inicialmente, a interface de exibição era feita através de planilhas do Excel. Sua utilização neste formato foi importante durante o desenvolvimento inicial do projeto, pois atuou como prova de conceito. No entanto, a exigência de uma interface compartilhada entre diversos usuários e com uma quantidade enorme de informação, inviabilizava a continuação das planilhas. Dessa forma, buscou-se uma solução em ambiente WEB na rede local da AMT. A tela de login da plataforma pode ser vista na Figura 4. Todos os usuários precisam estar cadastrados no sistema para efetuar o acesso. Não existem custos adicionais para o cadastro de novos usuários e este acesso pode ser feito através de qualquer computador conectado na rede corporativa da empresa.
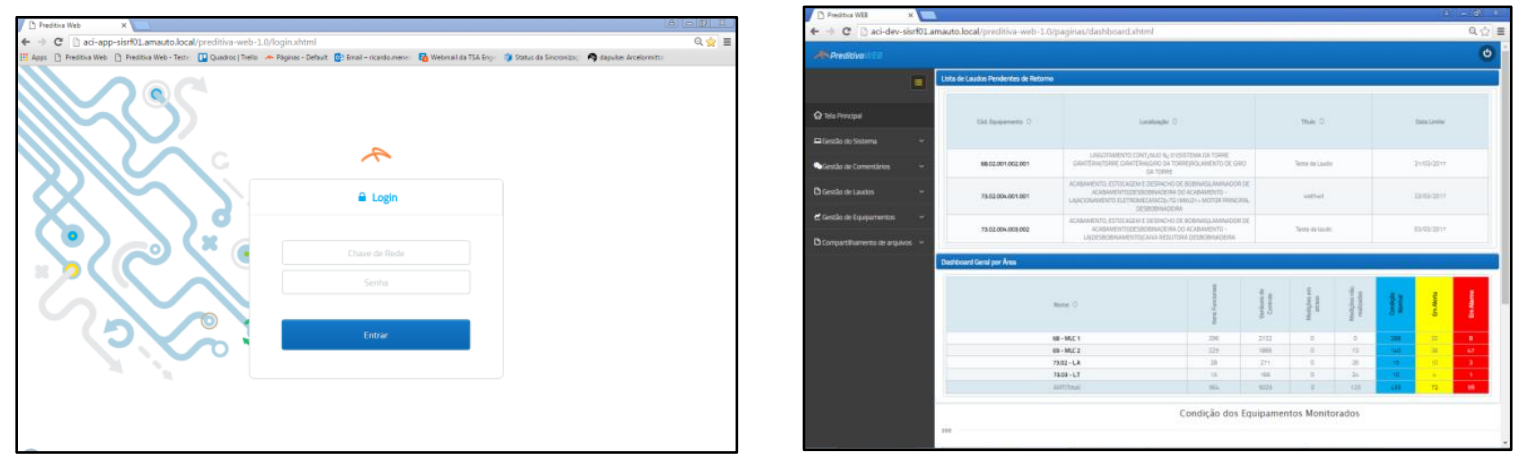

Figura 4 - Tela de Login da PreditivaWEB

Figura 5 - Tela inicial com Dashboard na PreditivaWEB

\section{1- Principais Funcionalidades}

Neste tópico serão abordadas algumas das funcionalidades desenvolvidas na Preditiva Web (interface de integração). Como foi mencionado anteriormente, buscase uma plataforma para fazer gestão dos equipamentos em alarme, levantamento de indicadores da manutenção preditiva de forma otimizada, entre outros itens.

\section{2- $\quad$ Painel de Indicadores}

Depois de efetuado o login, o sistema apresenta em sua tela inicial um Painel de Indicadores que traz informações gerais das áreas cadastradas. Dessas informações podemos citar alguns dados estatísticos de manutenção (KPIs) como variáveis em alarme, itens com medições em atraso, número de medições não realizadas, entre outras. Ainda nesta mesma tela é possível estratificar todos os ativos cadastrados com variáveis de controle alarmadas. É possível visualizar a tela inicial com o Dashboard. 


\section{3- Histórico de Equipamentos}

A funcionalidade mais importante do sistema pode ser vista a aba de Gestão de Equipamentos. Este espaço foi destinado para apresentação do histórico dos ativos cadastrados na plataforma. Todas as inspeções e informações desses equipamentos, oriundas dos diversos sistemas utilizados na AMT, podem ser acessadas e visualizadas através de tabelas, gráficos de tendência de uma ou mais variáveis (Figura 6).

A navegação pelos ativos é feita por meio de uma árvore de equipamentos que segue a mesma organização e estrutura do sistema CMMS da AMT. Foram utilizadas diferentes cores para indicar na árvore os equipamentos que estiverem alarmados. A árvore de navegação pode ser vista na Figura 6
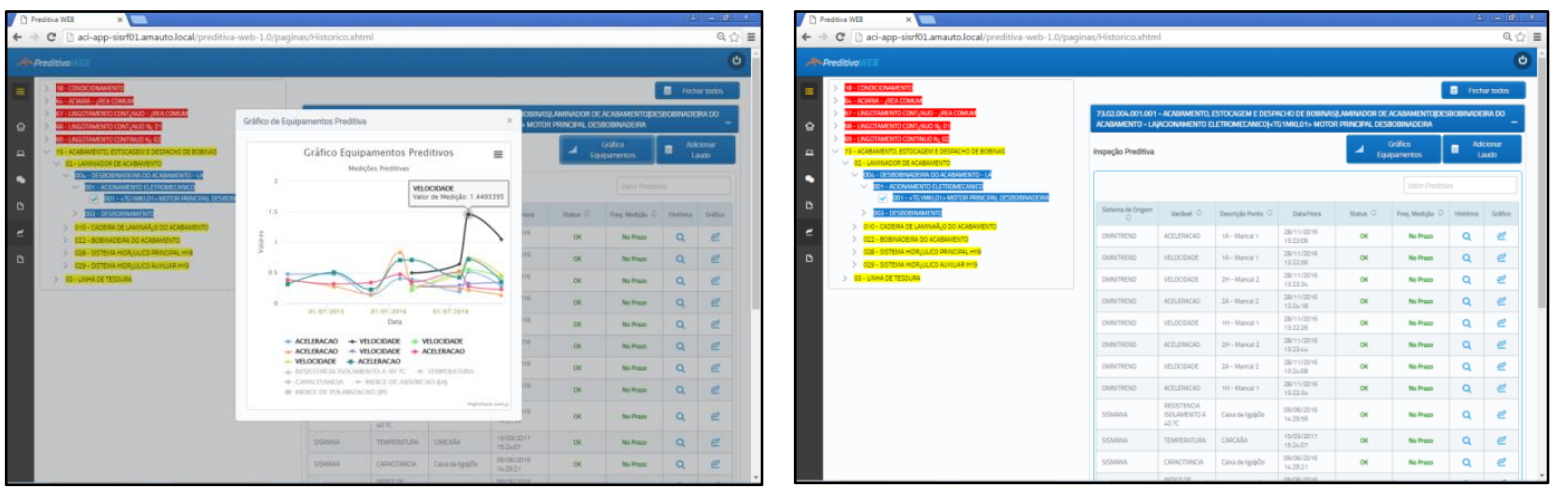

Figura 6 - Gráfico do Equipamento com exibição de diversas variáveis e Figura 7 - Tela com a Árvore de navegação dos equipamentos e Tabela de variáveis do Equipamento

\section{4- Laudos}

Durante o estudo do projeto, o grupo verificou que algumas informações importantes sobre o histórico dos equipamentos eram perdidas devido à falta de registro dessa informação. Muitas das analises realizadas pelos especialistas acabavam perdidas em planilhas e trocas de e-mail. Dessa forma, era preciso criar alguma ferramenta para documentar estas análises, suas respectivas ações e resultados, até para verificar a qualidade dessas ações.

Assim, foi desenvolvida uma funcionalidade para gestão de laudos. Os Laudos são documentos com a finalidade de ajudar as diferentes equipes de manutenção durante suas tomadas de decisão. Esses laudos são criados pelos especialistas da área de apoio de manutenção da AMT, sob demanda, com o intuito de comentar sobre a condição do equipamento e fazer recomendações sobre as ações necessárias. Também foram criadas formas efetivas de comunicação e controle desses laudos. Durante a criação de um laudo é possível enviar um e-mail notificando os responsáveis sobre a existência desse novo laudo. Para que o laudo não seja esquecido, o responsável é novamente notificado caso não dê um retorno sobre suas 
ações perante aquele laudo. Todo laudo possui um campo de preenchimento para este retorno. Dessa forma, é possível que o especialista acompanhe as ações e resultados sobre suas recomendações. Além disso, todos os laudos ficam salvos para futuras consultas. Na Figura 7 é possível visualizar a tela de criação de um laudo.

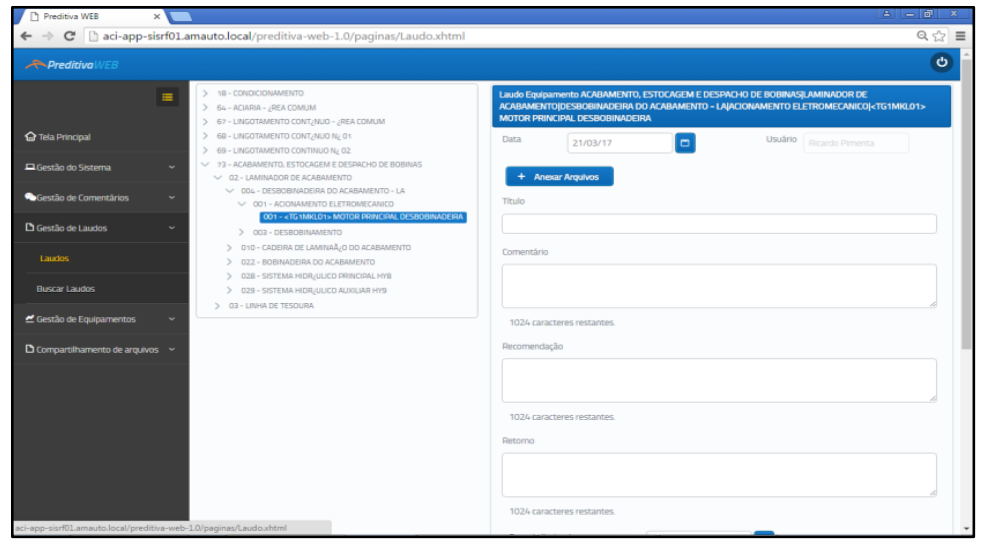

Figura 7 - Tela de criação de Laudos.

\section{4- RESULTADOS}

A integração das informações de inspeções trouxe não somente facilidade de consultas e correlação de variáveis dos ativos para facilidade de diagnóstico e análise de condição e de desempenho dos ativos. Permitiu também o registro de histórico das variáveis e de cada ativo, laudos detalhados e um controle minucioso de cada equipamento.

\section{orientação sobre disponibilização de recursos do PDA Caterpilar c/ GESMan}

... O Sistema de Inspeção Integrada desenvolvido na ArcelorMittal Tubarão vem atender uma antiga necessidade das equipes de inspeção e manutenção da ArcelorMittal Tubarão, no que diz respeito a facilidade de consulta e correlação das variáveis de controle dos ativos, pois permite visualizar os resultados das inspeções em uma única plataforma com todas as variáveis de controle dos ativos, onde as exceções são facilmente detectadas..

O custo do sistema foi significantemente reduzido quando comparado às plataformas comercias existentes no mercado.

O fato de o sistema ser desenvolvido internamente permite seu continuo desenvolvimento, e futuras melhorias e funcionalidades já estão planejadas como podemos citar: A identificação automatizada de gravidade dos alarmes, baseado em pontuação e peso por variável de acordo com o tipo de ativo, e mais a longo prazo uma plataforma complementar mais robusta que auxilie na tomada de decisão das equipes de manutenção (support decision) baseado em filtros e dados mais apurados das variáveis. 


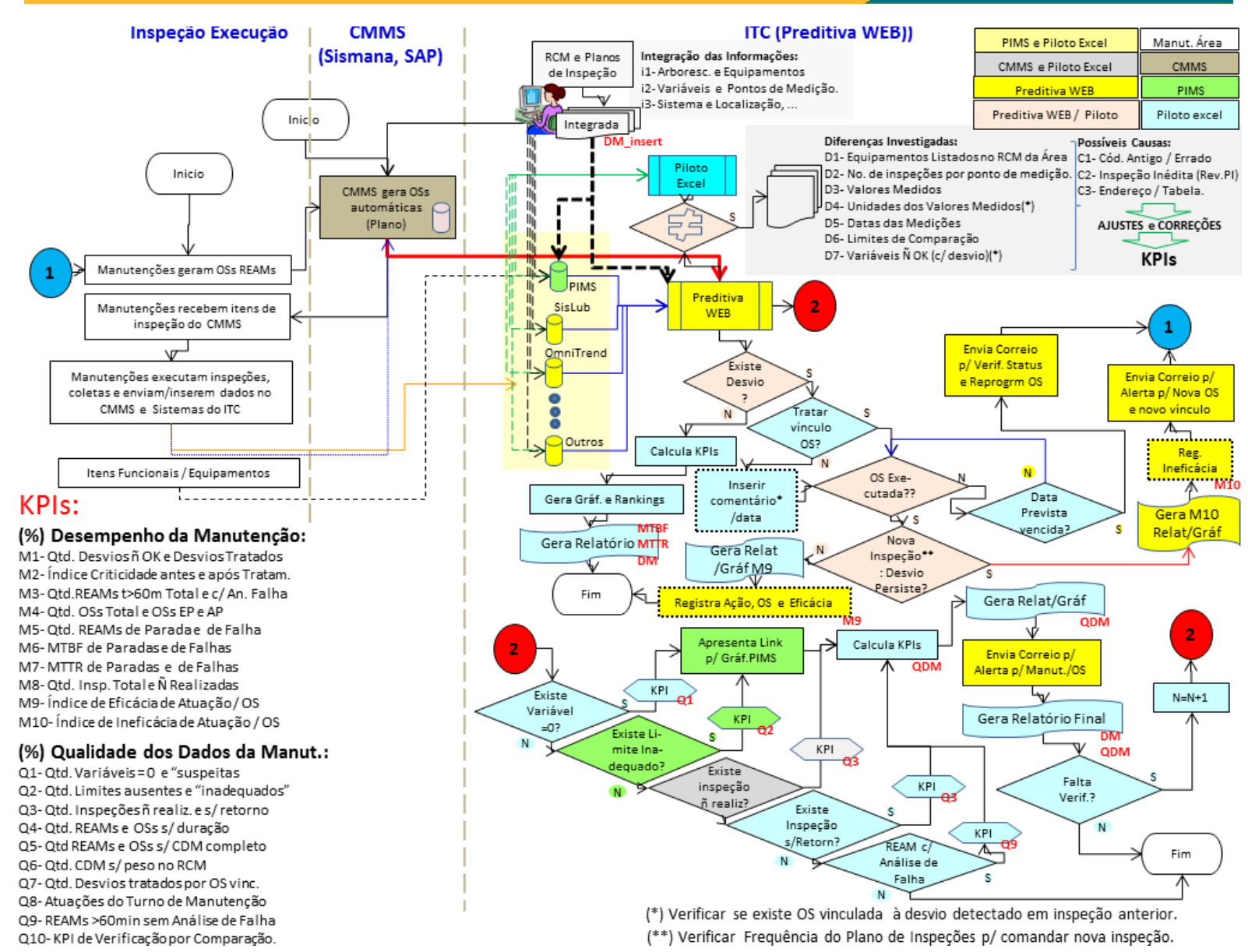

Figura 8. Diagrama de Integração, Processos e Fluxos de Informações.

\section{REFERÊNCIAS}

.1- ABRAMAN. Página eletrônica: <http://www.abraman.org.br/>. Acesso em 11 abr. 2017.

.2- ALMEIDA, M. T. Manutenção Preditiva: Confiabilidade e Qualidade. 2000. Disponível em: 〈http://www.mtaev.com.br/download/mnt1.pdf>. Acesso em 06 out. 2011.

.3- ASSOCIAÇÃO BRASILEIRA DE NORMAS TÉCNICAS. NBR-5462: confiabilidade e mantenabilidade. Rio de Janeiro: ABNT, 2004. 